\title{
Effects of flavonol-rich green tea (Camellia sinensis L. cv. Sofu) on blood glucose and insulin levels in diabetic mice
}

\author{
Sachiko Nomura $^{1 *}$, Manami Monobe ${ }^{1}$, Kaori Ema ${ }^{1}$, Akiko Matsunaga $^{1}$, Mari Maeda-Yamamoto ${ }^{2}$ and Hideki Horie $^{1}$ \\ ${ }^{1}$ Tea Industry Research Division, NARO Institute of Vegetable and Tea Science, 2769 Kanaya-Shishidoi, Shimada, Shizuoka 428-8501, Japan \\ ${ }^{2}$ Food Function Division, NARO National Food Research Institute, 2-1-12 Kan-nondai, Tsukuba, Ibaraki 305-8642, Japan
}

\begin{abstract}
Findings from epidemiological studies and intervention trials seem to suggest that green tea consumption prevents type 2 diabetes. The anti-diabetic effects are most commonly attributed to the polyphenolic fraction of green tea. Green teas contain catechins; however, they also contain flavonols and their glycosides. Here, we compared between a common and a flavonol-rich tea cultivar, with regard to the anti-diabetic effects, in a type 2 diabetes mouse model using NSY (Nagoya-ShibataYasuda) mice. Water or a tea infusion, made from a common (control tea, TeaC) or a flavonol-rich (flavonol-rich tea, TeaF) cultivar, was given to the NSY mice as the solo drinking fluid from 4 until 13 weeks of age. At 12 weeks of age, fasting plasma insulin concentration was significantly lower in mice drinking TeaF than in mice drinking water. Relative to water, TeaC had no significant effect on plasma insulin levels. Thus, there were significant differences in efficacy with regard to maintenance of glucose homeostasis between a common and a flavonol-rich tea cultivars; therefore, tea flavonols are likely to have benefits with regard to preventing diabetes.
\end{abstract}

\section{Introduction}

Diabetes is serious public health issue, and its related conditions pose a growing and potentially enormous economic burden. Although effective surgical and pharmacological methods have been developed to treat symptoms related to diabetes, these treatments can be costly and are not without potential adverse effects [1-4]. The development of dietary agents for the prevention of diabetes could represent a cost-effective and safe approach to the public health problem. Epidemiological findings have indicated that green tea consumption may prevents type 2 diabetes, non-insulin dependent diabetes mellitus $5,6]$, and mounting evidence shows that green tea consumption improves glucose metabolism in human [7] and diabetic mice [8]. Green tea contains characteristic polyphenolic compounds, catechins which are thought to be primarily responsible for the physiological effects of green tea [9]. However, flavonols and their glycosides are also present in tea [10], and quercetin, one of the major tea flavonols, also has anti-diabetic effects in mouse models of diabetes [11-13]. We previously measured flavonol glycoside concentrations in tea infusions from various cultivars and identified cultivars with high flavonol contents [14]. Here, we compared the anti-diabetic effects between a common tea cultivar and a flavonol-rich cultivar to examine whether tea flavonols can ameliorate glucose intolerance in a mouse model of type 2 diabetes. Previously, NSY (Nagoya-Shibata-Yasuda) mice were generated by selective ICR mouse colony to establish a mouse model of type 2 diabetes [15]; these mice spontaneously develop renal lesions similar to diabetic nephropathy, but do not become severe obesity at any age of development [16]. These characteristics are similar to the pathophysiologic features of human type 2 diabetes patients; therefore, NSY mice were used as a model of type 2 diabetes in the present study.

\section{Materials and methods}

NSY mice (4-week-old, male) were purchased from Hoshino Lab Animals Co. (Ibaraki, Japan). The mice were given a standard laboratory diet (CRF-1, Charles River Laboratories Inc. Wilmington, $\mathrm{MA})$ in an environmentally controlled room $\left(24^{\circ} \mathrm{C}, 60 \%\right.$ relative humidity, a 12-h light/dark cycle).This study was approved by the Ethical Committee on Animal Experiments at the NARO Institute of Vegetable and Tea Science (No.H26-01), and all animal experiments were performed by following Law no. 105 and Notification no. 6 of the government of Japan.

'Sofu' green tea leaves (Flavonol-rich Tea: TeaF) were obtained from the NARO plantation in Makurazaki, Kagoshima, Japan, and control 'Yabukita' tea leaves (Control Tea: TeaC) were obtained from the NARO plantation in Kanaya, Shizuoka, Japan. The dried green tea leaves of each cultivars were steeped separately in cold water for one hour; aliquots of the filtrates were frozen until used in experiments. The stock solutions were diluted with water just before being given to mice and fresh drinking fluid was provided to all mice every two days. LC/MS was used to measure flavonol glycoside levels in the diluted tea infusions as described previously [14]. HPLC was used to measure catechin and caffeine levels as described previously [17].

Correspondence to: Sachiko Nomura, Tea Quality and Function Research Group, Tea Industry Research Division, National Institute of Vegetable and Tea Science, National Agriculture and Food Research Organization, 2769 Kanaya-Shishidoi, Shizuoka, 428-8501 Japan, Tel: +81-547-45-4101; Fax: +81-0547-46-2169, E-mail: nonnon7@affrc.go.jp

Key words: flavonol-rich tea cultivars, quercetin glycosides, glucose tolerance test, NSY diabetic mice

Received: June 12, 2015; Accepted: July 22, 2015; Published: July 25, 2015 
To investigate whether either tea infusion could affect glucose intolerance, TeaCand TeaF were given to NSY mice as the solo drinking fluid (TeaC and TeaF groups, respectively) for 8 weeks. Control NSY mice were given water (Water group) instead of tea infusions. Body weight and food consumption were measured once and twice a week, respectively. Glucose tolerance tests (GTTs) were performed by intraperitoneally injecting glucose $(2 \mathrm{~g} / \mathrm{kg}$ body weight $)$ into mice that had fasted overnight and had been drinking the experimental fluids for 4 or 8 weeks; these animals were 8 or 12 weeks of age, respectively. Blood samples were obtained from the tail vein at $0,30,60,120$ and $240 \mathrm{~min}$ after the glucose injection. Blood glucose concentration was measured using a Glucose Pilot system (Iwai Chemical Co., Tokyo, Japan). For measurement of insulin, whole blood was collected from the tail vein at 0 and $30 \mathrm{~min}$ during i.p. GTT. Each blood sample was immediately centrifuged $\left(3,000 \mathrm{xg}, 5 \mathrm{~min}, 4^{\circ} \mathrm{C}\right)$ to separate the plasma. Libs Mouse Insulin ELISA Kits (Shibayagi Co., Ltd., Gunma, Japan) were used according to the manufacturer's protocol to measure plasma insulin levels. At the end of the experiment, the mice were 13 weeks of age; they fasted overnight and were then anesthetized with isoflurane. A blood sample was collected into a heparinized tube from the infraaxillary artery of each anesthetized mouse. The pancreas, liver, and epididymal fat pads were quickly removed from each animal and weighed. Triglyceride Test Wako kits (Wako Pure Chemical Industries, Ltd., Osaka, Japan) were used to measure plasma triglyceride levels enzymatically.

All data obtained are expressed as the mean \pm SEM. One-way factorial analysis of variance (ANOVA) and subsequent Scheffe's post-hoc test were used to compare means among groups. When data were not normally distributed, the Kruskal-Wallis test was applied. Differences with a $p$ value less than 0.05 were considered significant. Notably, to determine the significance of the effects of tea infusions on the blood glucose and insulin levels (Figure 1), repeated measures ANOVA was performed, and then, if statistical significance was detected by this analysis, further statistical comparisons among the groups were conducted for each measurement time by one-way ANOVA and subsequent Scheffe's tests.
A

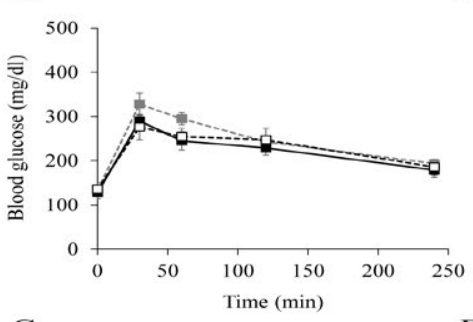

C

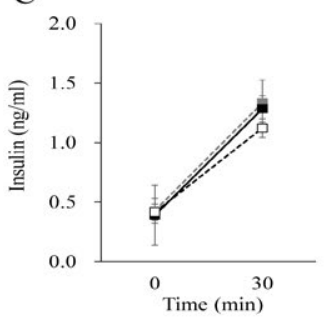

B

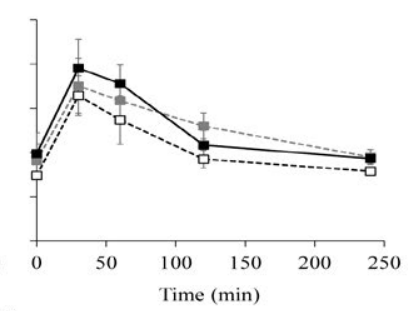

D

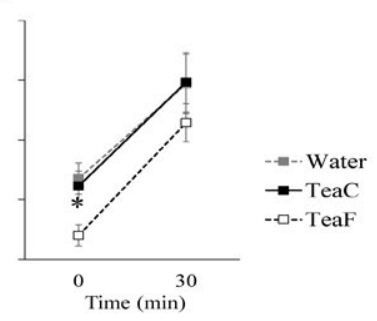

Figure 1. Effects of tea infusion on glucose tolerance and insulin response in NSY mice. Glucose tolerance tests (GTTs) were performed at $8(\mathrm{~A}, \mathrm{C})$ or 12 (B, D) weeks of age. Blood glucose concentration (A, B) and plasma insulin (C, D) were measured during GTTs. All values are mean $\pm \operatorname{SEM}(\mathrm{n}=4$ or 5$) .{ }^{*} p<0.05$; significantly different from the Water group at each time-point, determined by one-way ANOVA, followed by Scheffé's test.

\section{Results and discussion}

Using protocols from our previous study [14], we determined the concentrations of two myricetin, five quercetin and three kaempferol glycosides in each type of tea infusion (Table 1). Among the measured quercetin glycosides, quercetin-3-O-glucosyl-rhamnosyl-glucoside was the most abundant in both tea infusions, TeaC and TeaF, as was the case in the previous study [14]. The level of quercetin-3-O-glucosylrhamnosyl-glucoside was notably higher in TeaF than in TeaC. Total aglycone content was also higher in TeaF than in $\mathrm{TeaC}$ for all of the aglycones measured. As shown in Table 2, while there were minor variations in the composition of catechins between $\mathrm{TeaC}$ and $\mathrm{TeaF}$, the total amounts of catechins and caffeine in TeaF were similar to those in TeaC. Daily intakes of total flavonolaglycones in TeaC and TeaF groups were approximately700 and $1500 \mu \mathrm{g}$, respectively. For both tea infusion groups, daily intake of total catechins and caffeine were approximately $7.0 \mathrm{mg}$ and $1.3 \mathrm{mg}$, respectively.

There were no significant differences among all groups with regard to body weight or food intake throughout the experiment (Table 3). Thus, relative to water, neither TeaC nor TeaF significantly affected body weight gain or food intake of NSY mice. The results of GTTs are shown in Figure 1. In the Water group, insulin response to glucose at 12 weeks of age (Figure $1 \mathrm{~B}$ and $1 \mathrm{D}$ ) was impaired relative to that at 8 weeks of age (Figure 1A and 1C). The result is consistent with previous findings that showed NSY mice spontaneously develop diabetes in an

Table 1. Flavonol glycoside levels in tea infusions.

\begin{tabular}{|l|c|c|}
\hline Flavonol Glycosides $(\boldsymbol{\mu g} / \mathbf{m l})$ & TeaC & TeaF \\
\hline Myricetin-3- $O$-glucoside & 13.0 & 20.7 \\
\hline Myricetin-3- $O$-galactoside & 29.8 & 44.6 \\
\hline Quercetin-3- $O$-glucoside (Isoquercitrin) & n.d & 3.4 \\
\hline Quercetin-3- $O$-galactoside (Hyperoside) & 6.1 & 15.1 \\
\hline Quercetin-3- $O$-rutinoside (Rutin) & 1.4 & 20.6 \\
\hline Quercetin-3-O-glucosyl-rhamnosyl-glucoside & 93.4 & 325.9 \\
\hline Quercetin-3- $O$ - glucosyl-rhamnosyl-galactoside & 80.0 & 90.4 \\
\hline Kaempferol-3-O-rutinoside & 2.5 & 10.4 \\
\hline Kaempferol-3-O-glucosyl-rhamnosyl-glucoside & 36.4 & 93.4 \\
\hline Kaempferol-3- $O$-glucosyl-rhamnosyl-galactoside & 96.1 & 73.2 \\
\hline Total Aglycones $(\boldsymbol{\mu g}$ /ml) & & \\
\hline Myricetin & 28.4 & 43.3 \\
\hline Quercetin & 72.6 & 185.2 \\
\hline Kaempferol & 51.3 & 68.1 \\
\hline
\end{tabular}

Control Tea (TeaC) was made from a common tea cultivar, and Flavonol-rich Tea (TeaF) was made from a flavonol-rich tea cultivar. Total aglycone content was calculated from the concentrations of the corresponding glycosides. n.d.: not detected.

Table 2. Catechin and caffeine levels in tea infusions.

\begin{tabular}{|l|c|c|}
\hline Catechins (mg/100 ml) & TeaC & TeaF \\
\hline Catechin & 1.9 & 3.2 \\
\hline Catechin gallate & n.d & 0.2 \\
\hline Gallocatechin & 2.9 & 4.4 \\
\hline Gallocatechin gallate & n.d & n.d \\
\hline Epicatechin & 16.6 & 25.4 \\
\hline Epicatechin gallate & 5.5 & 5.4 \\
\hline Epigallocatechin & 81.8 & 78.8 \\
\hline Epigallocatechin gallate & 42.5 & 26.2 \\
\hline Total & 151.2 & 143.6 \\
\hline Caffeine (mg/100 ml) & $\mathbf{3 0 . 7}$ & $\mathbf{2 3 . 8}$ \\
\hline
\end{tabular}

n.d: not detected 
Table 3. Changes in body weight and food intake.

\begin{tabular}{|c|c|c|c|}
\hline & Water & TeaC & TeaF \\
\hline \multicolumn{4}{|l|}{ Body weight (g) } \\
\hline $4 w$ & $22.08 \pm 1.08$ & $21.46 \pm 0.52$ & $21.36 \pm 0.38$ \\
\hline $8 w$ & $33.98 \pm 1.21$ & $33.76 \pm 0.40$ & $32.70 \pm 0.63$ \\
\hline $12 w$ & $36.48 \pm 1.47$ & $35.70 \pm 0.62$ & $35.04 \pm 0.39$ \\
\hline Daily food intake (g/day/head) & $4.31 \pm 0.10$ & $4.62 \pm 0.18$ & $4.52 \pm 0.13$ \\
\hline
\end{tabular}

All values are mean $\pm \operatorname{SEM}(\mathrm{n}=4$ or 5$)$. There were no significant between-group differences in the body weight or food intake throughout the experiment.

Table 4. Effects of tea infusion on body weight, organ weight, and plasma triglyceride level in NSY mice.

\begin{tabular}{|l|c|c|c|}
\hline & Water & TeaC & TeaF \\
\hline Body weight $(\mathrm{g})$ & $32.83 \pm 1.17$ & $32.98 \pm 0.53$ & $32.34 \pm 0.36$ \\
\hline Pancreas $(\mathrm{mg})$ & $169.1 \pm 7.0$ & $168.5 \pm 5.4$ & $172.4 \pm 7.4$ \\
\hline Liver $(\mathrm{g})$ & $1.250 \pm 0.029$ & $1.298 \pm 0.043$ & $1.254 \pm 0.034$ \\
\hline Epididymal fat pad $(\mathrm{mg})$ & $1006.2 \pm 135.3$ & $726.6 \pm 54.5$ & $647.6 \pm 48.8 *$ \\
\hline Plasma triglyceride $(\mathrm{mg} / \mathrm{dl})$ & $135.0 \pm 15.6$ & $149.6 \pm 9.3$ & $119.7 \pm 16.0$ \\
\hline
\end{tabular}

At 13 weeks of age, animals were denied food overnight and then subject to these assays in a fasting state. All values are mean $\pm \operatorname{SEM}(\mathrm{n}=4$ or 5$)$. ${ }^{*} p<0.05$; significantly different from the Water group, determined by one-way ANOVA, followed by Scheffé's test.

age-dependent manner $[16,18]$. At 12 weeks of age, although glycemic excursions during the GTT were not significantly different among groups (Figure 1B), fasting plasma insulin concentration of the TeaF group was significantly lower than that of the Water group (Figure 1D). Fasting plasma insulin concentration of the TeaC group did not differ significantly from that of the Water group (Figure 1D). These result indicated that consumption of TeaF, but not TeaC, could improve the impaired insulin response in the NSY mice.

At the end of the experimental period, with the mice at 13 weeks of age and in a fasting state, there were no significant differences among the groups with regard to body weight, pancreatic weight, or liver weight (Table 4). However, epididymal fat pad weights were significantly less in the TeaF group than in the Water group. Mean fat pad weights in the TeaC group were also less than those in the Water group, however this difference was not significant $(p<0.1)$. The previous studies found mild obesity in the NSY mice $[16,18]$, and mean fat pad mass of the Water group was greater than that of age-matched outbred ICR mice ( $484.1 \pm 60.6 \mathrm{mg}, \mathrm{n}=4$, unpublished data), from which the NSY mice were selectively bred for glucose intolerance. Therefore, these tea infusions, especially TeaF, are thought to suppress the fat accumulation in NSY mice. Numerous studies demonstrate that green tea and its extracts have anti-obesity effects in mouse models of diabetes $[8,19]$. For each of the three treatment groups of the NSY mice, fasting plasma triglyceride levels were distinctly higher than those of the agematched ICR mice $(85.5 \pm 9.0 \mathrm{mg} / \mathrm{dl}, \mathrm{n}=4$, unpublished data) (Table 4). However, for NSY mice, there were no significant differences in the triglyceride levels among the three treatment groups (Water, TeaC, and TeaF); suggesting that TeaC and TeaF did not affect plasma triglyceride levels in NSY mice.

In the present study, we found that there were significant differences between a common and a flavonol-rich tea cultivars with regard to glucose homeostasis in NSY mice, and that tea flavonols are likely to have benefits for preventing diabetes. Further studies on tea flavonols and diabetes are warranted; such as those designed to obtain direct evidence of higher plasma levels of flavonols and their derivatives following consumption of flavonol-rich teas and examining the mechanisms in which flavonols improve insulin sensitivity in type 2 diabetic mouse models.

\section{Acknowledgement}

We would like to express our sincere gratitude to Drs. Atsushi Nesumi and Katsuyuki Yoshida of the NARO Institute of Vegetable and Tea Science for providing the tea leaf samples.

\section{References}

1. Graham DJ, Ouellet-Hellstrom R, MaCurdy TE, Ali F, Sholley C, et al. (2010) Risk of acute myocardial infarction, stroke, heart failure, and death in elderly Medicare patients treated with rosiglitazone or pioglitazone. JAMA 304: 411-418. [Crossref]

2. Pories WJ (2008) Bariatric surgery: Risks and rewards. J Clin Endocrinol Metab 93 S89-96. [Crossref]

3. Poitou Bernert C, Ciangura C, Coupaye M, Czernichow S, Bouillot JL, et al. (2007) Nutritional deficiency after gastric bypass: diagnosis, prevention and treatment. Diabetes Metab 33: 13-24. [Crossref]

4. Filippatos TD, Derdemezis CS, Gazi IF, Nakou ES, Mikhailidis DP, et al. (2008) Orlistat-associated adverse effects and drug interactions: a critical review. Drug Saf 31: 53-65. [Crossref]

5. Song Y, Manson JE, Buring JE, Sesso HD, Liu S (2005) Associations of dietary flavonoids with risk of type 2 diabetes, and markers of insulin resistance and systemic inflammation in women: a prospective study and cross-sectional analysis. $J \mathrm{Am} \mathrm{Coll}$ Nutr 24: 376-384. [Crossref]

6. Iso H, Date C, Wakai K, Fukui M, Tamakoshi A; JACC Study Group (2006) The relationship between green tea and total caffeine intake and risk for self-reported type 2 diabetes among Japanese adults. Ann Intern Med 144: 554-562. [Crossref]

7. Nagao T, Meguro S, Hase T, Otsuka K, Komikado M, et al. (2009) A catechin-rich beverage improves obesity and blood glucose control in patients with type 2 diabetes. Obesity (Silver Spring) 17: 310-317. [Crossref]

8. Sae-tan S, Grove KA, Lambert JD (2011) Weight control and prevention of metabolic syndrome by green tea. Pharmacol Res 64: 146-154. [Crossref]

9. Khan N, Mukhtar H (2013) Tea and health: studies in humans. Curr Pharm Des 19: 6141-6147. [Crossref]

10. Sakakibara H, Honda Y, Nakagawa S, Ashida H, Kanazawa K (2003) Simultaneous determination of all polyphenols in vegetables, fruits, and teas. J Agric Food Chem 51: 571-581. [Crossref]

11. Kobori M, Masumoto S, Akimoto Y, Takahashi Y (2009) Dietary quercetin alleviates diabetic symptoms and reduces streptozotocin-induced disturbance of hepatic gene expression in mice. Mol Nutr Food Res 53: 859-868. [Crossref]

12. Jeong SM, Kang MJ, Choi HN, Kim JH, Kim JI (2012) Quercetin ameliorates hyperglycemia and dyslipidemia and improves antioxidant status in type 2 diabetic $\mathrm{db} /$ db mice. Nutr Res Pract 6: 201-207. [Crossref]

13. Zhang R, Yao Y, Wang Y, Ren G (2011) Antidiabetic activity of isoquercetin in diabetic KK -Ay mice. Nutr Metab (Lond) 8: 85. [Crossref]

14. Monobe M, Nomura S, Ema K, Matsunaga A, Nesumi A, et al. (2015) Quercetin glycosides-rich tea cultivars (Camellia sinensis L.) in Japan. Food Sci Technol Res 21: 333-340.

15. Fushimi H, Shibata M, Tarui S (1980) Glycosidase activities in the liver and kidney of hereditary diabetic mice. J Biochem 87: 941-949. [Crossref]

16. Ueda H, Ikegami H, Yamato E, Fu J, Fukuda M, et al. (1995) The NSY mouse: a new animal model of spontaneous NIDDM with moderate obesity. Diabetologia 38: 503508. [Crossref]

17. Maeda-Yamamoto M, Ema K, Monobe M, Tokuda Y, Tachibana H (2012) Epicatechin3-O-(3"-O-methyl)-gallate content in various tea cultivars (Camellia sinensis L.) and its in vitro inhibitory effect on histamine release. J Agric Food Chem 60: 2165-2170. [Crossref]

18. Ueda H, Ikegami H, Kawaguchi Y, Fujisawa T, Nojima K, et al. (2000) Age-dependent changes in phenotypes and candidate gene analysis in a polygenic animal model of Type II diabetes mellitus; NSY mouse. Diabetologia 43: 932-938. [Crossref]

19. Babu PV, Liu D, Gilbert ER (2013) Recent advances in understanding the anti-diabetic actions of dietary flavonoids. J Nutr Biochem 24: 1777-1789. [Crossref]

Copyright: (C)2015 Nomura S. This is an open-access article distributed under the terms of the Creative Commons Attribution License, which permits unrestricted use, distribution, and reproduction in any medium, provided the original author and source are credited. 